\title{
Estudo do perfil epidemiológico da vacinação contra o HPV no Estado do Piauí
}

\author{
Study of the epidemiological profile of the vaccination against HPV in the State of Piauí \\ Estudio del perfil epidemiológico de la vacunación contra VPH en el Estado de Piauí
}

Recebido: 02/11/2021 | Revisado: 09/11/2021 | Aceito: 16/11/2021 | Publicado: 26/11/2021

\author{
Amanda Nogueira Araújo \\ ORCID: https://orcid.org/0000-0002-7053-3422 \\ Centro Universitário Santo Agostinho, Brasil \\ E-mail: amandan0@hotmail.com \\ Emilly Valéria Ribeiro Coelho \\ ORCID: https://orcid.org/0000-0003-2545-0465 \\ Centro Universitário Santo Agostinho, Brasil \\ E-mail:emillyvaleriaribeiro@hotmail.com \\ Maria Helena Rodrigues Mesquita Britto \\ ORCID: https://orcid.org/0000-0002-0673-836X \\ Centro Universitário Santo Agostinho, Brasil \\ E-mail: mhrmesquita@hotmail.com
}

\begin{abstract}
Resumo
Objetivo: Avaliar o perfil epidemiológico da vacinação contra o HPV no estado do Piauí. Metodologia: O estudo desse trabalho foi por meio de uma pesquisa exploratória, epidemiológica e descritiva com abordagem transversal, quantitativa e retrospectiva. Os dados epidemiológicos coletados foram referentes ao Estado do Piauí e a coleta foi realizada entre março e abril de 2021, a partir do banco de dados do Departamento de Informática do Sistema Único de Saúde (DATASUS). Foram incluídos dados de indivíduos com idades entre 9 e 26 anos, sexo feminino e masculino e que receberam a vacina quadrivalente contra o HPV entre os anos de 2017 a 2020 . Resultados e Discussão: Os indivíduos do sexo masculino com faixa etária de 11 anos foram os que apresentaram maior número de vacinação $(33,5 \%)$, enquanto a faixa de 17 anos $(0,1 \%)$ a menor número de vacinação, a primeira dose foi de $53 \%$ no sexo feminino e $61,9 \%$ no sexo masculino, obtendo uma aplicação que na segunda dose que foi $46,8 \%$ no sexo feminino e $37 \%$ no sexo masculino. Considerações finais: Desta forma, percebe-se que o estado do Piauí não obteve uma procura significativa e satisfatória em relação à vacina HPV no intervalo temporal alvo deste estudo. Apesar de a região Nordeste ter sido a segunda região do país que mais vacinou contra o HPV, o Piauí não ficou em uma boa posição durante esse período, e consequentemente não atingiu a meta estipulada pelo Ministério da Saúde para cada ano.
\end{abstract}

Palavras-chave: Epidemiologia; HPV; Vacina; Saúde pública.

\begin{abstract}
Objective: To evaluate the epidemiological profile of vaccination against HPV in the state of Piauí. Methodology: The study of this work was through an exploratory, epidemiological and descriptive research with a transversal, quantitative and retrospective approach. The epidemiological data collected were related to the State of Piauí and the collection was carried out between Marchand April 2021, from the data base of the Information Technology Department of the Unified Health System (DATASUS). Data from individuals aged between 9 and 26 years old, female and male and Who received the quadrivalent vaccine against HPV between 2017 and 2020 were included. Results and Discussion: Males aged 11 years were those with the highest number of vaccinations (33.5\%), while the 17 -year-old group $(0.1 \%)$ had the lowest number of vaccinations, the first dose was $53 \%$ in females and $61.9 \%$ in males, obtaining an application that in the second dose was $46.8 \%$ in females and $37 \%$ in males. Final considerations: In this way, it is clear that the state of Piauí did not obtain a significant and satisfactory demand for the HPV vaccine in the target period of this study. Although the Northeast region was the second region in the country that vaccinated the most against HPV, Piauí was not in a good position during this period, and consequently did not reach the target stipulated by the Ministry of Health for each year.
\end{abstract}

Keywords: Epidemiology; HPV; Vaccine; Public health.

\section{Resumen}

Objetivo: Evaluar el perfil epidemiológico de la vacunación contra el VPH en el estado de Piauí. Metodología: El estudio de este trabajo se realizó mediante una investigación exploratoria, epidemiológica y descriptiva con enfoque transversal, cuantitativo y retrospectivo. Los datos epidemiológicos recolectados se relacionaron con el estado de Piauí y la recolección se realizó entre marzo y abril de 2021, a partir de la base de datos del Departamento de Tecnología de La Información del Sistema Único de Salud (DATASUS). Se incluyeron datos de individuos de entre 9 
y 26 años, mujeres y hombres, que recibieron la vacuna tetravalente contra el VPH entre 2017 y 2020 . Resultados y Discusión: Los hombres de 11 años fueron los que presentaron mayor número de vacunaciones (33,5\%), mientras que el grupo de 17 años $(0.1 \%)$ tuvo el menor número de vacunaciones, la primera dosis fue 53\% en mujeres y $61.9 \%$ en hombres, obteniendo una aplicación que en la segunda dosis fue $46.8 \%$ en mujeres y $37 \%$ en hombres .Consideraciones finales: De esta manera, es evidente que el estado de Piauí no obtuvo una demanda significativa y satisfactoria de la vacuna contra el VPH en el período objetivo de este estudio. Si bien la región Nordeste fue la segunda región del país que más vacunó contra el VPH, Piauí no estuvo en una buena posición durante este período, por lo que no alcanzó la meta estipulada por El Ministerio de Salud para cada año.

Palabras clave: Epidemiología; VPH; Vacuna; Salud pública.

\section{Introdução}

O HPV (papilomavírus humano) é um vírus que infecta pele ou mucosas. são listados mais de 100 tipos de HPV, sendo que desses, 15 tipos como o 16, 18, 31, 33, 45, 58 e outros, apresentam malignidade de alto risco. O vírus HPV acomete homens e mulheres podendo surgir lesões na região genital e extragenital, evidenciando manifestações clínicas, subclínicas e latentes (Costa; Goldenberg, 2013). A transmissão do HPV pode ocorrer pelo contato direto dos órgãos genitais durante a prática sexual sem proteção, que ocasiona a penetração do vírus no tecido epiteliais através de microabrasões. Outra forma de transmissão é o contato direto ou indireto com as lesões localizadas em outras regiões do corpo e além disso, pode ocorrer também a transmissão vertical durante a gestação ou parto (Taquaryet al., 2018).

A infecção ocorre por meio da penetração do vírus na célula do hospedeiro com liberação de seu DNA e consequente replicação. O vírus pode provocar manifestações clínicas e subclínicas ou perdurar em estado latente por vários anos Abreu et al., 2018). Nos casos de infecção por HPV em que há manifestações clínicas pode-se observar o surgimento de verrugas e lesões, popularmente denominadas de "crista de galo", podendo apresentar tamanhos variados e aspecto de couve-flor. Em mulheres, essas lesões podem surgir na vagina, vulva, região pubiana, perianal, ânus e colo do útero. Nos homens podem aparecer no pênis, bolsa escrotal, região pubiana, perianal e anus. Além disso, as lesões também podem surgir em outras regiões do corpo como boca e garganta (Libera et al., 2016). De acordo com Abreu et al. (2018) a presença de lesões verrugosas ou granulares e únicas ou múltiplas, permite a realização do diagnóstico clínico em homens e mulheres.

O tratamento em casos de infecção por HPV inclui a remoção de verrugas, quando existentes, por meio de cauterização. Além disso, para controlar a infecção e evitar complicações, a abordagem mais efetiva de acordo com o Ministério da Saúde, continua sendo o rastreamento através do exame preventivo de Papanicolau. Desde 1940 constitui o método de triagem e prevenção para as lesões precursoras do câncer do colo uterino que mais atingem as mulheres. Este exame sofreu modificações na sua realização, é um teste efetivo, de baixo custo e considerado a melhor estratégia para reduzir a incidência desse tipo de câncer. Em relação à prevenção do contágio do vírus, tem-se disponível vacinas bivalentes e quadrivalentes, o uso de preservativos que servem como barreiras durante a relação sexual e a higiene íntima (Costa; Goldenberg, 2013; Brasil, 2013).

As vacinas profiláticas contra o HPV trouxeram a possibilidade de ações em nível primário, já que a prevenção só ocorria em nível secundário. É uma estratégia utilizada em alguns países desde a sua aprovação em junho de 2006 (Borsatto; Vidal; Rocha, 2011). No Brasil e em vários países, existem no mercado duas vacinas aprovadas para uso na população: a vacina bivalente Cervarix ${ }^{\circledR}$ que é eficaz contra os vírus 16 e 18 e a vacina tetravalente ou quadrivalenteGardasil@ (guardianofsquamousintraepiteliallesion) que protege contra os tipos 6, 11, 16 e 18 (Calumbyet al., 2020). Assim, por meio do Programa Nacional de Imunizações, o Ministério da Saúde, ampliou em 2014 o Calendário Nacional de Vacinação do Adolescente com a introdução da vacina quadrivalente contra o HPV no Sistema Único de Saúde (SUS) como estratégia de saúde pública, em mulheres de 9 a 13 anos, com objetivo de reforçar as atuais ações de prevenção do câncer do colo de útero. Esta faixa etária foi escolhida porque é nessa idade que a vacina tem a melhor resposta (Brasil, 2013). 
A partir de 2017 a vacinação foi ampliada para meninas de 9 a 14 anos e meninos de 11 a 14 anos também foram introduzidos como público alvo, ambos com os esquemas de duas doses (0 e 6 meses). Além disso, homens e mulheres com idade entre 9 e 26 anos vivendo com HIV/Aids, situação de órgãos sólidos e medula óssea transplantados e pacientes oncológicos passaram a ser contemplados com o esquema de três doses (0, 2 e 6 meses) (Brasil, 2018).

Apesar de serem realizadas várias campanhas educativas e preventivas para realizar a vacinação contra o HPV, ainda se percebe uma elevada prevalência dessa infecção viral no Brasil (Silva et al., 2014). Diante disso, percebe-se a necessidade de traçar de forma descritiva, o perfil epidemiológico do público alvo a fim de gerar informações que permitam realizar um melhor aprimoramento das ações de prevenção, e consequentemente, a redução no número de casos da infecção por HPV. Assim, o presente trabalho tem como objetivo avaliar o perfil epidemiológico da vacinação contra o HPV no estado do Piaú́.

\section{Metodologia}

Este estudo consiste em uma pesquisa exploratória, epidemiológica e descritiva com abordagem transversal, quantitativa e retrospectiva. A pesquisa exploratória com caráter descritivo, tem como objetivo proporcionar o conhecimento do assunto estudado de forma mais profunda, buscando descrever as características de determinada população ou fenômeno relacionando suas variáveis. Além disso, a aplicação da abordagem quantitativa em uma pesquisa, permite quantificar, descobrir e classificar as relações dos dados obtidos (Oliveira Júnior, 2017). Em relaçãoao caráter epidemiológico com delineamento transversal, o objetivo é estudar uma população específica estimando a frequência de um determinado fenômeno de saúde (Bastos; Duquia, 2007).

Os dados epidemiológicos coletados foram referentes ao Estado do Piaú e a coleta foi realizada entre março e abril de 2021, a partir do banco de dados do Departamento de Informática do Sistema Único de Saúde (DATASUS). Foram incluídos dados de paciente com idades entre 9 e 26 anos, sexo feminino e masculino e que receberam a vacinaquadrivalente contra o HPV entre os anos de 2017 a 2020. Além disso, foram excluídos os seguintes dados: vacinação fora do período estabelecido, vacinação de outras patologias e pacientes que não apresentavam a faixa etária estabelecida.

Durante a coleta de dados foram utilizados dados secundários do Sistema de Informação do Programa Nacional de Imunizações (SI-PNI). Os dados obtidos foram tabulados em planilhas no Excel, para melhor organização e compreensão, e posteriormente foram analisados de acordo com parâmetros estatísticos através do Software PRISM 9.0.2.

Por se tratar de dados de seguimento público em plataformas online, não foi necessário submeter o projeto ao Comitê de Ética em Pesquisa (CEP), respeitando-se a Resolução 466/12 do Conselho Nacional de Saúde (CNS), que estabelece as Diretrizes e Normas Regulamentadoras de Pesquisas Envolvendo Seres Humanos.

\section{Resultados}

No Estado do Piauí, durante o período entre 2017-2020, foram aplicadas 322.161 doses da vacina contra HPV, sendo que por sexo, 165.028 foram aplicadas em mulheres e 157.133 foram aplicadas em homens. De acordo com a Tabela 1 , para o sexo feminino o total de doses aplicadas foi de 49.855 (30\%) em 2017, 35.067 (21\%) em 2018, 35.495 (22\%) em 2019 e 44.611 (27\%) em 2020. Pode-se observar que 2017 apresentou a maior taxa de vacinação para o sexo feminino. Já em 2018 e 2019, houve um decaimento de $9 \%$ e $8 \%$, respectivamente. Em 2020, a cobertura vacinal voltou a aumentar em comparação com os dois anos anteriores. Em relação ao sexo masculino, apresentado na Tabela 2, observa -se que um total de 51.261 $(33 \%)$ doses foram aplicadas em 2017, 40.859 (26\%) em 2018, 32.115 (20,5\%) em 2019 e 32,898 (21\%) em 2020. Nota-se que a cobertura vacinal em homens foi diminuindo a cada ano durante esse período. Com base nesses resultados, verifica-se que a cobertura vacinal em homens e mulheres não atingiu a meta de $80 \%$ estipulada pelo Ministério da Saúde para o público alvo. 
Tabela 1. Cobertura da vacina HPV quadrivalente do sexo feminino no Piauí, de 2017 a 2020.

\begin{tabular}{ll}
\hline Ano & Feminino (\%) \\
\hline $\mathbf{2 0 1 7}$ & $49.855(30 \%)$ \\
$\mathbf{2 0 1 8}$ & $35.067(21 \%)$ \\
$\mathbf{2 0 1 9}$ & $35.495(22 \%)$ \\
TOTAL & $44.611(27 \%)$ \\
\hline
\end{tabular}

Fonte: Dados a Pesquisa (2021).

Tabela 2. Cobertura da vacina HPV quadrivalente do sexo masculino no Piauí, de 2017 a 2020.

\begin{tabular}{ll}
\hline Ano & Masculino (\%) \\
\hline $\mathbf{2 0 1 7}$ & $51.261(33 \%)$ \\
$\mathbf{2 0 1 8}$ & $40.859(26 \%)$ \\
$\mathbf{2 0 1 9}$ & $32.115(20 \%)$ \\
TOTAL & $32.898(21 \%)$ \\
\hline
\end{tabular}

Fonte: Dados a Pesquisa (2021).

As tabelas 1 e 2, permitem uma melhor compreensão no que se refere à adesão do grupo feminino e masculino, respectivamente, em relação a vacinação.

Gráfico 1. Curva da cobertura vacinal do sexo feminino no Piauí, de 2017 a 2020 (n=165.028).

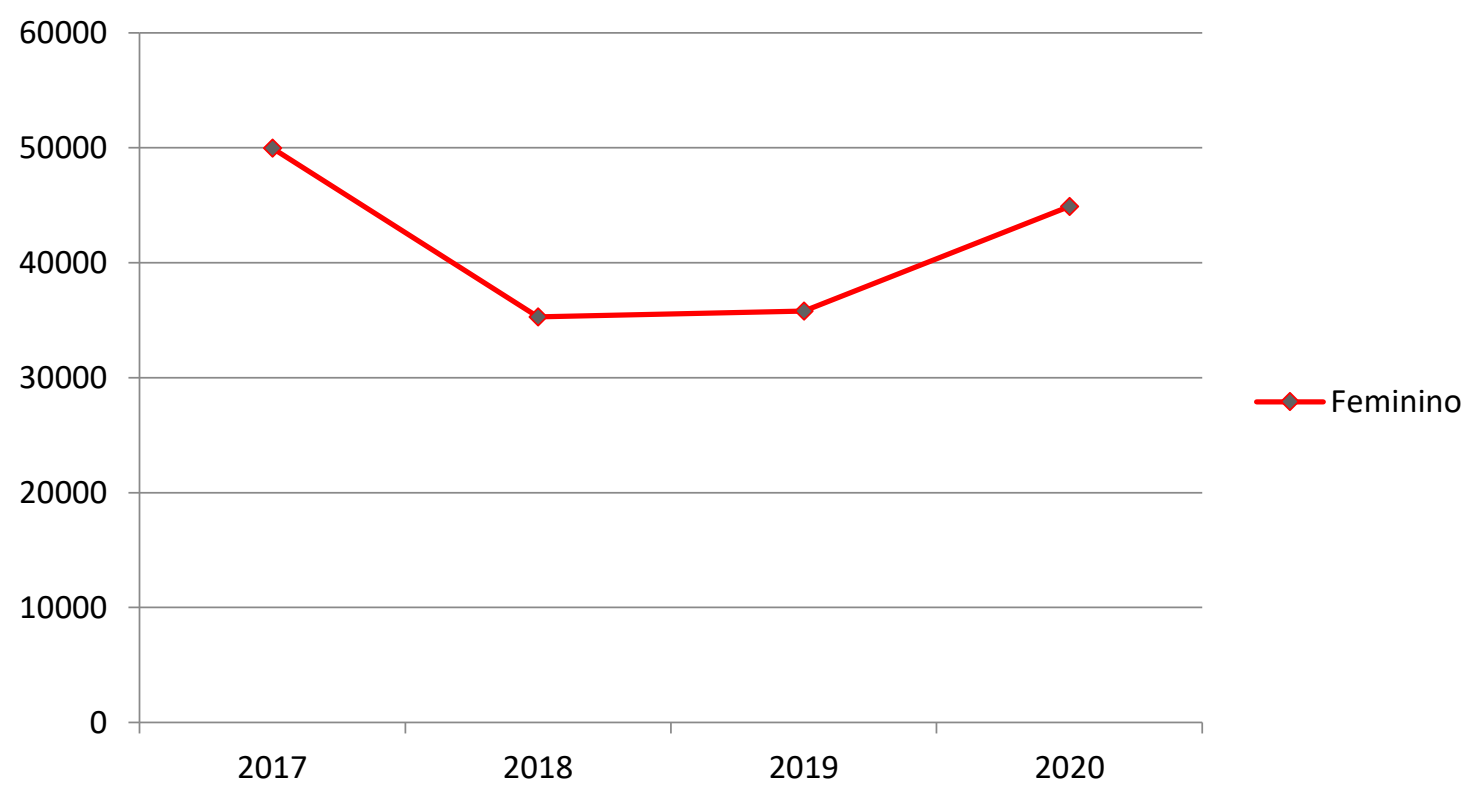

Fonte: Dados a Pesquisa (2021). 
Gráfico 2. Curva da cobertura vacinal do sexo feminino no Piauí, de 2017 a 2020 (n=157.133).

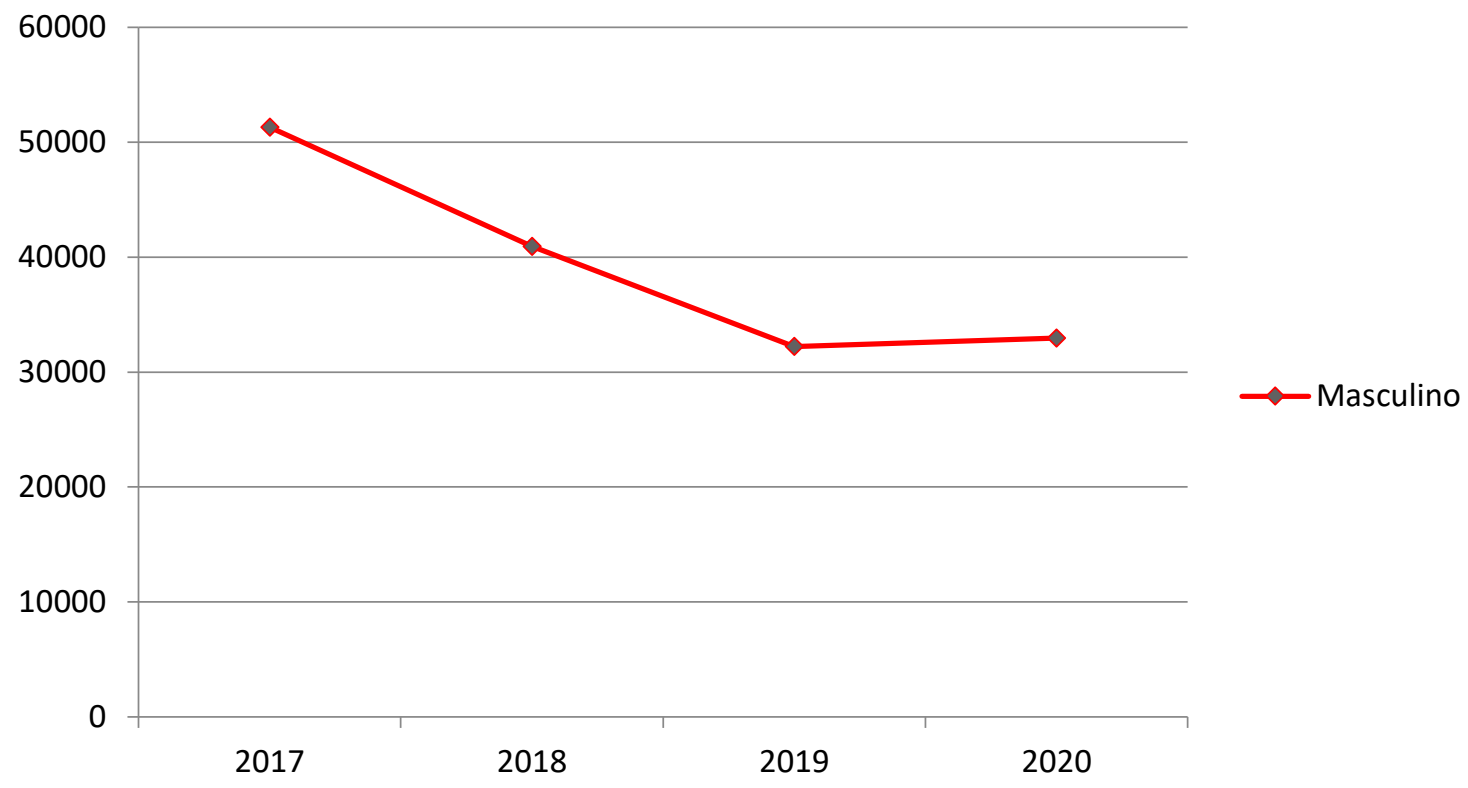

Fonte: Dados a Pesquisa (2021).

Evidenciou-se que o público feminino apresentou uma diminuição na taxa de vacinação, no entanto, estabilizou-se e progrediu. Em contrapartida, o público masculino apresentou constate queda com leve estabilização entre os anos 2019 e 2020.

Os resultados referentes às faixas etárias do sexo feminino vacinadas no Estado do Piauí, apresentados na Tabela 3 , revelam que a maior taxa de vacinação ocorreu em crianças de 9 anos (45,5\%) e a menor taxa ocorreu em mulheres de 17 anos.

Tabela 3. Cobertura da vacina HPV quadrivalente do sexo feminino, por idade, no Piauí, de 2017 a 2020. (n=165.028).

\begin{tabular}{ll}
\hline Faixa etária & Feminino $(\boldsymbol{\%})$ \\
\hline 9 & $75.739(45,5 \%)$ \\
10 & $38.436(23 \%)$ \\
11 & $21.503(13,1 \%)$ \\
12 & $12.632(7,6 \%)$ \\
13 & $7.997(4.8 \%)$ \\
15 & $4.829(2,4 \%)$ \\
16 & $1.122(0,7 \%)$ \\
17 & $230(0,1 \%)$ \\
19 & $209(0,1 \%)$ \\
$20-26$ & $228(0,1 \%)$ \\
TOTAL & $233(0,1 \%)$ \\
\hline
\end{tabular}

Fonte: Dados a Pesquisa (2021).

Na Tabela 4 estão distribuídos os números de doses aplicadas por faixa etária no sexo masculino entre 2017-2019 no Estado do Piauí. Constata -se maior vacinação em relação a faixa etária de 11 anos $(33,5 \%)$ enquanto a faixa de 17 anos $(0,1 \%)$ se destacou como a que menos recebeu vacinações. 
Tabela 4. Cobertura da vacina HPV quadrivalente do sexo masculino, por idade, no Piauí, de 2017 a 2020. (n=157.456).

\begin{tabular}{ll}
\hline Faixa etária & Masculino $(\boldsymbol{\%})$ \\
\hline 9 & $1.471(0,9 \%)$ \\
10 & $2.955(1,9 \%)$ \\
11 & $52.822(33,5 \%)$ \\
12 & $44.704(28,4 \%)$ \\
14 & $31.438(19,9 \%)$ \\
15 & $18.564(11,8 \%)$ \\
16 & $3.747(2,4 \%)$ \\
17 & $244(0,2 \%)$ \\
19 & $140(0,1 \%)$ \\
$20-26$ & $108(0,09 \%)$ \\
TOTAL & $83(0,05 \%)$ \\
\hline
\end{tabular}

Fonte: Dados a Pesquisa (2021).

Os dados apresentados na Tabela 5 apresentam os resultados referentes ao sexo masculino e feminino em relação às doses das vacinações contra o HPV no estado do Piauí.

Tabela 5. Doses aplicadas e cobertura da vacina HPV quadrivalente, em ambos os sexos, no Piauí, de 2017 a 2020.

\begin{tabular}{clcc}
\hline Dose & $\begin{array}{l}\text { HPV Quadrivalente }- \\
\text { Feminino }(\boldsymbol{\%})\end{array}$ & $\begin{array}{c}\text { HPV Quadrivalente- } \\
\text { Masculino (\%) }\end{array}$ & TOTAL \\
\hline $\mathbf{1}^{\mathbf{a}}$ dose & $87.391(53 \%)$ & $97.999(61,9 \%)$ & 185.390 \\
$\mathbf{2}^{\mathbf{a}}$ dose & $76.879(46,8 \%)$ & $58.776(37 \%)$ & 135.655 \\
$\mathbf{3}^{\mathbf{a}}$ dose & $748(0,2 \%)$ & $358(0,1 \%)$ & 1.116 \\
TOTAL & 165.028 & 157.133 & 322.161 \\
\hline
\end{tabular}

Fonte: Dados a Pesquisa (2021).

Levando em consideração o sexo masculino, notou-se que a primeira dose obteve maior número de vacinas com 97.999 representando $61,9 \%$, ao passo que a segunda dose apresentou 580.776, representando $37 \%$ das vacinas, e a terceira dose com 358 vacinações, representando $0,1 \%$. Observou-se em relação ao sexo feminino que a primeira dose obteve o maior número de vacinações, com 87.391 , sendo representados por $53 \%$ do total de vacinações, enquanto que a segunda dose obteve 76.879 vacinações, sendo representadas com 46,8\% das vacinas, e a terceira dose com 748 vacinações, representando $0,2 \%$ das vacinas.

Em relação à cobertura total, a primeira dose apresentou 185.390 aplicações, a segunda dose foi aplicada 35.655 vezes e a terceira dose 1.116 vezes. Destaca-se que a terceira dose apresenta uma menor taxa de aplicações por conta do público alvo não ser tão grande comparado aos demais grupos.

Apesar de não estar representado em tabela ou gráfico, outro resultado importante diz respeito à região Nordeste que, durante esse período de 4 anos, foi a segunda região do país que mais vacinou contra o HPV com 27,9\% das vacinações, ficando atrás da região Sudeste representando $41,1 \%$ da cobertura vacinal no país. Além disso, dentre os 9 estados da região Nordeste, o estado que mais vacinou contra o HPV foi a Bahia com 21,3\% das vacinas. O Piauí, no entanto, ficou na sétima posição representando apenas $5,5 \%$ da cobertura vacinal contra o HPV. 


\section{Discussão}

A partir de 2014, período da inclusão da vacina HPV no Calendário Nacional de Imunização, o Programa Nacional de Imunizações do Ministério da Saúde, vem gerando ações direcionadas para o alcance da meta de cobertura vacinal de $80 \%$ na população alvo. Para tal, estão sendo efetuadas parcerias com as sociedades científicas e trabalho conjunto as igrejas, organizações não-governamentais e com a mídia (Brasil, 2017). Apesar disso, a meta não foi atingida no Estado do Piauí no período de 2017-2020. Durante esse período a maior cobertura vacinal ocorreu no ano de 2017, atingindo 30\% e 33\% no sexo feminino e masculino, respectivamente. Em relação a faixa etária, o público feminino e masculino de 9 anos foram os mais vacinados.

Tais achados se assemelham com o estudo de Ferreira et al. (2018), no município de Princesa Isabel - PB em 2018, sobre a vacinação contra o HPV, segundo o qual a vacinação atingiu cerca de 45\% da população do sexo feminino de 9 a 12 anos. Esses resultados também se entram em concordância com os estudos de Machado e Alcântara (2016) no município Rolim de Moura - GO em 2015, que aponta a vacinação de meninas contra HPV atingindo cerca de 43,75\% da população alvo que era de 9 a 12. Esses estudos também evidenciam que a maior procura pela vacina foi realizada por meninas com faixa etária de 9 anos correspondendo a 50,57\% no próprio município.

De acordo com Brasil (2018), diversos estudos comprovam que a vacinação contra o HPV deve ser realizada na faixa etária de 9 a 14 anos, pelo fato de a vacinação provocar nesse período uma maior produção de anticorpos em comparação a produção natural pelo vírus HPV. Além disso, esse período também é considerado como um dos melhores momentos para a vacinação pelo fato de ser antes do início da vida sexual ativa e os pais ainda preservarem a prática de levar os filhos para tomar outras vacinas. É importante ressaltar que o público masculino foi inserido no esquema de vacinação para diminuir os riscos de infecção em mulheres e consequente câncer de colo de útero. Essa medida faz com que as taxas de infeção oral por HPV diminuam significativamente.

No que diz respeito as doses, tanto no público feminino quanto no masculino, a primeira dose foi mais prevalente em comparação com a segunda dose. Outros municípios brasileiros de diferentes estados, como Itajaí-GO (Silva et al.,2020), Amparo-SP (Ruas et al., 2017), Conceição do Araguaia-PA (Cunha et al., 2018), Princesa Isabel-PB (Ferreira et al., 2018) e Volta Redonda-RJ (Cabral et al., 2019) baixa na cobertura da segunda dose da vacina contra o HPV. França et al. (2017) também evidenciou que cerca de 16 municípios da Microrregião de Serra Geral em MG, atingiram a cobertura para a primeira dose, mas houve queda significativa na aplicação da segunda dose. Diante disso, possivelmente vários adolescentes ficaram com o esquema vacinal incompleto.

Diversos fatores são pontados como dificultadores para adesão à vacina como: o medo dos eventos adversos entre os adolescentes e familiares (França et al., 2017 e Cabral et al., 2019), a pouca informação e esclarecimento a respeito da importância da vacina por parte dos adolescentes e responsáveis (Ruas et al., 2017; Cunha et al., 2018; França et al., 2019 e Cabral et al., 2019), a dificuldade de diálogo entre os pais e filhos sobre sexualidade (Franca et al., 2017; Cabral et al., 2019), a mudança da estratégia de vacinação utilizando apenas as Unidades Básicas de Saúde e reduzindo a participação das escolas como locais de vacinação (França et al., 2017; Pereira; Souza, 2017 e Manganelliet al., 2018), o medo da iniciação precoce da atividade sexual e o despreparo dos profissionais de saúde (França et al., 2017).

Avelino, Rodrigues e Souza (2018) relatam que os fatores mais frequentes para a baixa adesão a vacinação contra o HPV são o medo de reações adversas causadas pela vacina e a impossibilidade de deslocamento até uma unidade básica de saúde para se vacinar. Em relação às reações adversas, há vários estudos científicos que garantem a segurança da vacina quadrivalente tendo em vista que não foram encontradas evidências de efeitos colaterais graves ocorrendo de forma significativa em pessoas que tomaram a vacina. 
Outro importante fator que deve ser citado, diz respeito às campanhas antivacinas. De acordo com Pereira (2018) esses movimentos têm crescido significativamente nos últimos anos e estão relacionados à diminuição de adesão a vacina, sendo atribuída ao alto números de efeitos adversos. $\mathrm{O}$ autor também ressalta que o indivíduo que participa de movimentos como esse, mesmo possuindo a liberdade de não se vacinar, ocasiona consequências negativas na população em geral ao induzir as pessoas a também não se vacinarem.

Diante dos resultados encontrados nesse estudo, destaca-se a importância da continuidade de campanhas em escolas, igrejas, mídia e outras instituições ou meios, com a finalidade de esclarecer questões sobre o vírus HPV e suas consequências, bem como elucidar a necessidade da vacinação contra o HPV.

\section{Considerações Finais}

Ao final desta pesquisa percebe-se que o estado do Piauí não obteve uma procura significativa e satisfatória em relação à vacina HPV no intervalo temporal alvo deste estudo. Apesar de a região Nordeste ter sido a segunda região do país que mais vacinou contra o HPV, o Piauí não ficou em uma boa posição durante esse período, e consequentemente não atingiu a meta estipulada pelo Ministério da Saúde para cada ano.

Em suma, é possível verificar que promovendo educação em saúde, com palestras e o Programa Saúde na Escola, tirando as dúvidas e levando informações ao público alvo, assim como também aos responsáveis pelas adolescentes, resultará em uma ampliação na cobertura vacinal.

Tais resultados são importantes para mostrar como se encontra a vacinação contra o HPV no município do estudo comparado com os níveis de vacinação a níveis nacionais e regionais, desta forma, subsidiando novos estudos na área e dados relevantes para população e saúde pública do país.

\section{Referências}

Abreu, M. S. N. et al. (2018). Conhecimento e percepção sobre o HPV na população com mais de 18 anos da cidade de Ipatinga, MG, Brasil. Ciênc. saúde colet., 23(3), 849-860. https://www.scielosp.org/article/csc/2018.v23n3/849-860/

Avelino, J. P. et al. (2021). Análise da vacinação contra o hpv em uma capital do nordeste do brasil. Revista uningá, 58, eUJ3572. <http://revista.uninga.br/index.php/uninga/article/view/3572.

Bastos, J. L. D.; \& Duquia, R. P. (2007). Um dos delineamentos mais empregados em epidemiologia: estudo transversal. Scientia Medica, 17(4), 229-232. https://www.google.com/url?sa=t\&source=web\&rct=j\&url=https://revistaseletronicas.pucrs.br/ojs/index.php/scientiamedica/article/download/2806/7864/\&ved =2ahUKEwjVioWP3dbvAhWeIrkGHUsyAYYQFjABegQIBBAC\&usg=AOvVaw3QOwCIfP_uUkb-f5c49yqo\&cshid=1617063488889.

Bergmann, A.; Casado, L.; \& Thuler, L. C. S. (2012). Perfil das Pacientes com Câncer do Colo do Útero no Brasil, 2000-2009: Estudo de Base Secundária. Revista Brasileira de Cancerologia.58, 351-357. https://rbc.inca.gov.br/revista/index.php/revista/article/view/583

Borsatto, A. Z.; Vidal, M. L. B.; \& Rocha, R. C. N. P. (2011). Vacina contra o HPV: Aspectos Práticos. Rev Brasil Cancerol. 7(1), 67-74. https://rbc.inca.gov.br/revista/index.php/revista/article/download/690/462/\#: :text=Demonstrou\%20que\%20a\%20vacina\%20\%C3\%A9,para\%20cada\%20tipo $\% 20$ espec\%C3\%ADfico5\%2C\%2021.

Brasil. Ministério da Saúde. Secretária de Vigilância em Saúde. Departamento de Vigilância das Doenças Transmissíveis. Coordenação Geral Do Programa Nacional de Imunização. (2013). Informe técnico da ampliação da oferta das vacinas papilomavírus humano 6,11,16 e 18 (recombinante) - vacina HPV quadrivalente e meningocócica C (conjugada). https://www.google.com/url?sa=t\&source=web\&rct=j\&url=https://portalarquiv os2.saude.gov.br/image s/pdf/2018/marco/14/Informe-T--cnico-HPV-MENINGITE.pdf\&ved=2ahUKEwjWqPT6npPzAhWdlZUCHUChA2kQFnoECAYQAQ\&usg=AOv Vaw1RAb UpUjq 7I-1FvcDkgklF.

Brasil. Ministério da Saúde. Secretária de Vigilância em Saúde. Departamento de Vigilância das Doenças Transmissíveis. Coordenação Geral Do Programa Nacional de Imunização. (2013). Informe técnico sobre a vacina contra o papilomavírus humano (HPV). https://portalarquivos2.saude.gov.br/images/pdf/2015/junho/26/Informe-T--cnico-Introdu----o-vacina-HPV-18-2-2014.pdf

Brasil. Ministério da saúde. Saúde amplia vacinação de HPV para homens e mulheres até 26 anos, 2017. http://portalsaude.saude.gov.br/index.php/cidadao/principal/agencia-saude/29280- saude-amplia-vacinacao-de-hpv-para-homens-e-mulheres-ate-26-anos

Cabral, A. A. et al. (2019). Análise quantitativa da imunização contra o HPV no âmbito do Sistema Único de Saúde em um município Sul Fluminense. Brazilian Journal of Surgery and Clinical Research, 27(3). 42-46. https://www.mastereditora.com.br/download-3248

Calumby, R. J. N. et al. (2020). Papiloma Vírus Humano (HPV) e neoplasia cervical: importância da vacinação. BrasilianJournalof Health Review, 3(2), 1610-1628. https://www.brazilianjournals.com/index.php/BJHR/article/view/7486. 
Costa, L. A; Goldenberg, P. (2013). Papilomavírus Humano (HPV) entre Jovens: um sinal de alerta. Revista Saúde e Sociedade.22(1), 249-261. https://www.scielo.br/j/sausoc/a/JDV4DqKt5vjxxYDHSyYmRcJ/?lang=pt

Cunha, A. L. M.; Freire, K. S. \& Rocha, C. B. R. (2018). Adesão da vacinação contra o papiloma vírus humano (HPV) no município de Conceição do Araguaia-PA. Revista Científica Multidisciplinar Núcleo do Conhecimento. 15, 55-64. https://www.nucleodoconhecimento.com.br/saude/adesao-da-vacinacao

Ferreira, M. R. N. et al. (2018).Perfil epidemiológico da vacina HPV em um município do sertão paraibano do ano de $2016 . \quad F I P$, https://temasemsaude.com/wp-content/uploads/2018/10/fip201806.pdf.

França, S. B. F. et al. (2017). Adesão das adolescentes à campanha de vacinação contra o papiloma vírus humano: no Brasil, Minas Gerais e Microrregião da Serra Geral. Revista Unimontes Cientifica. 19(1), 11. https://www.periodicos.unimontes.br/index.php/unicientifica/article/view/2033

Libera, L. S. D. et al. (2016). Avaliação da infecção pelo Papiloma Vírus Humano (HPV) em exames citopatológicos. Revista RBAC. http://www.rbac.org.br/artigos/avaliacao-da-infeccao-pelo-papiloma-virus-humano-hpv-em-exames-citopatologicos-48-n2/.

Machado, P. A. T.; \& Alcântara, A. C. M. (2016). Cobertura vacinal contra o HPV em meninas de 9 a 11 anos no Município de Rolim de Moura - GO. Rev. Enfermagem e Saúde Coletiva, 1(2), 74-90. http://docplayer.com.br/39391179-Cobertura-vacinal-contra-o-hpv-em-meninas-de-09-a-11-anos-no-municipiode-rolim-de-moura-ro.html. Acesso em 10 mai. 2021.

Manganelli, L. A. G. et al. (2018). Avaliação da cobertura vacinal contra o Papilomavírus Humano a partir da vacina HPV quadrivalente recombinante nos

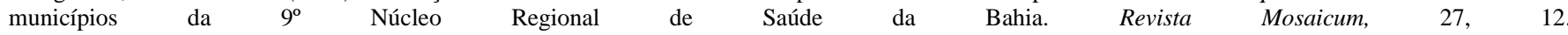
https://revistamosaicum.org/index.php/mosaicum/article/download/57/52/148

Oliveira Júnior, E. L. (2017). Pesquisa científica na graduação: um estudo das vertentes temáticas e metodológicas dos trabalhos de conclusão de curso. Trabalho de Conclusão de Curso (Graduação em Ciências Contábeis) - Universidade Federal de Uberlândia, [S. l.], 2017. https://repositorio.ufu.br/handle/123456789/20939.

Pereira, F. B.; \& Souza, E. P. (2017). Cobertura vacinal do HPV para adolescentes: desafios e possibilidades. Id onLine Rev. Mult. Psic. 11(38). https://idonline.emnuvens.com.br/id/article/view/918

Silva, D. S. M. et al. (2014). Rastreamento do câncer do colo do útero no Estado do Maranhão, Brasil. Revista Ciência e saúde coletiva. 19(4),11631170. https://www.scielo.br/j/csc/a/R9pz3PZSgVJXHF3WzQ9L4BF/abstract/?lang=pt

Silva, B. F. et al. (2020). O impacto da adesão vacinal contra o HPV entre jovens: um estudo transversal. BrazilianJournalofdevelopment, 6(8), 59080-59092. https://www.brazilianjournals.com/index.php/BRJD/article/view/15142/12493

Ruas, B. R. B., et al. (2017). Estratégia e adesão da vacinação contra HPV no município de Amparo, São Paulo. Revista Saúde em Foco, 9 , 11. https://portal.unisepe.com.br/unifia/wp-content/uploads/sites/10001/2018/06/008_estrategia_vacina\%C3\%A7\%C3\%A3o_hpv.pdf

Taquary, R. L. et al. (2018). Fatores de risco associados ao Papilomavírus Humano (HPV) e o desenvolvimento de lesões carcinogênicas no colo do útero: uma breve revisão. III CIPEEX - Ciência para a redução das desigualdades, 2, 855-859, 2018. Trabalho apresentado no III Congresso Internacional de Pesquisa, Ensino e Extensão. http://anais.unievangelica.edu.br/index.php/CIPEEX/article/view/3042\#: :text=Entre\%20os\%20fatores\%20de\%2 0risco,que $\% 20 \mathrm{o} \% 20$ indiv\%C3\%ADduo\%20se\%20encontra. 